\title{
Transfer Matrix Spectrum for O(N) High Temperature Lattice Classical Ferromagnetic Spin Systems and Staggering Transformations
}

\author{
Petrus H. R. dos Anjos* \\ Departamento de Física e Informática, IFSC-USP \\ C.P. 369, 13560-970 São Carlos, SP, Brazil \\ Paulo A. Faria da Veiga ${ }^{\dagger}$ and Michael $\mathrm{O}^{\prime}$ Carroll ${ }^{\ddagger}$ \\ Departamento de Matemática, ICMC-USP \\ C.P. $668,13560-970$ São Carlos SP, Brazil
}

PACS Numbers: 02.30.Tb,75.10.Hk,05.50.+q,11.10.St,03.65.Db.

Keywords: $\mathrm{O}(N)$ Spin System, Excitation Spectrum, Spectral Analysis, Bethe-Salpeter Equation.

July 16, 2003

\begin{abstract}
We obtain the low-lying energy-momentum spectrum for the imaginary-time lattice quantum field model associated with $d$-dimensional lattice ferromagnetic classical $N$-component vector spin systems at high temperature $(0<\beta \ll 1)$. Each system is characterized by a single site "a priori" spin probability distribution. The energy-momentum spectrum exhibits isolated dispersion curves which are identified as single particles and multi-particle bands. Our two-particle bound state analysis is restricted to a ladder approximation of the Bethe-Salpeter equation, and the existence of bound states depend on whether or not Gaussian domination for the four-point function is verified. It is known that two-particle bound states appear below the two-particle band if Gaussian domination does not hold. Here, we show that two two-particle bound states appear above the two-particle band if Gaussian domination is verified. We also show how the complete two-particle spectral pattern for these models can be understood by making a correspondence between the Bethe-Salpeter equation and a two-particle lattice Schrödinger Hamiltonian operator with attractive or repulsive spin-dependent delta potentials at the origin. A staggering transformation is used to relate the attractive and repulsive potential cases, as well as their associated Hamiltonians spectrum and eigenfunctions.
\end{abstract}

\section{Introduction and Results}

In recent papers [1]-[4], the low-lying energy-momentum (e-m) spectrum of the lattice quantum field theory associated with some lattice $\mathrm{O}(N)$ ferromagnetic classical spin models at high temperature $(\beta>0$ sufficiently

\footnotetext{
*Email: petrus@if.sc.usp.br

†Email: veiga@icmc.usp.br

${ }^{\ddagger}$ Email: ocarroll@icmc.usp.br
} 
small) is determined. The partition function for these $\mathrm{O}(N)$ ferromagnetic classical spin models is given formally by

$$
Z=\int e^{\beta \sum \vec{s}(x) \cdot \vec{s}(y)} \prod_{z \in \mathbb{Z}^{d}} e^{-V(|\vec{s}(z)|)} d \vec{s}(z),
$$

where $x=\left(x_{0}, \vec{x}\right) \in \mathbb{Z}^{d}, \vec{s}(x)=\left(s_{1}(x), s_{2}(x), \ldots, s_{N}(x)\right) \in \mathbb{R}^{N}$, and the sum is over unordered nearest neighbor pairs. $e^{-V(|\vec{s}(x)|)} d \vec{s}(x)$ is the single spin distribution (ssd). It is taken to be rotationally invariant and even. Also, $V(u)$ is assumed to be bounded from below and to grow to infinity at least quadratically. One important point about these $\mathrm{O}(N)$ models is that they involve an infinite number of degrees of freedom and do not correspond to perturbations about a Gaussian measure like we have for usual quantum field theories.

Concerning the e-m spectrum, it is found that there is a one-particle spectrum (lower mass gap), of multiplicity $N$, characterized by an isolated dispersion curve so that there is an upper mass gap as well. The asymptotic one-particle mass (i.e. the inverse correlation length) is of order $-\ln \beta$. Above the one-particle spectrum there are two-, three-,... particle bands, with finite width, and eventually overlapping for large particle number. The existence of a two-particle bound state spectrum near and below the two-particle band depends on the single spin distribution. We denote the infinite-volume, normalized single spin distribution moments by $\left\langle s_{i_{1}}\left(x_{1}\right) \ldots s_{i_{n}}\left(x_{n}\right)\right\rangle_{\beta}$, and $\langle\cdot\rangle$ stands for the $\beta=0$ averages, with all spins at the origin. Gaussian domination is characterized by the negative sign of the parameter

$$
\alpha_{N} \equiv\left\langle(\vec{s} . \vec{s})^{2}\right\rangle-\frac{N+2}{N}\langle\vec{s} . \vec{s}\rangle^{2} .
$$

Note that if the ssd is Gaussian, so that Wick's theorem applies, then $\alpha_{N}=0$. We call the case $\alpha_{N}>0$ Gaussian subjugation.

For the scalar case $N=1$ and Gaussian domination, there is no bound state below the two-particle threshold. However, if $\alpha_{N}>0$, it is shown that a two-particle bound state exists below and near the two-particle band. This result was obtained by considering a lattice version of the Bethe-Salpeter equation for these models. The analysis was carried out first at the level of a ladder approximation to the Bethe-Salpeter operator [1], and then it was extended to the complete models by controlling perturbations to this approximation [2]. The case $N>1$ was considered in [4] only within a ladder approximation to the Bethe-Salpeter equation, showing a richer structure for the two-particle bound states. For $N>1$, there are two bound states below the two-particle band in the Gaussian subjugation case. The one of lower mass has multiplicity one, such as non-degenerate, and the other has multiplicity $-1+N(N+1) / 2$.

More recently, for $N=1$, in [5] it was shown that one two-particle bound state exists above the two-particle band if Gaussian domination is satisfied.

The spectral pattern with a bound state below or above the two-particle band depends on Gaussian domination or subjugation. It can be understood by making the correspondence between a lattice version of the Bethe-Salpeter (B-S) equation, at zero system spatial momentum and in the ladder approximation, and the resolvent equation for a one-particle lattice Schrodinger Hamiltonian operator with a delta potential at the origin [6].

The free Hamiltonian is proportional to minus the lattice Laplacian $\Delta$ on the space $\ell_{2}\left(\mathbb{Z}^{d-1}\right)$. In this correspondence, for the $N=1$ model, the ladder approximation corresponds to a repulsive (attractive) $\delta$ potential at the origin if Gaussian domination (subjugation) holds.

As is well-known and physically intuitive, for the attractive case, there is a bound state below the continuum band. Surprisingly enough, for the repulsive case, there is a bound state above the band. At the physical level, these spectral phenomena can be understood by making the correspondence of the time-independent lattice Schrödinger eigenvalue equation with Newton's equations for polarized oscillations of an infinite classical massspring system with all masses equal, except for the one at the origin (isotopic-type defect), see [7]. Depending on whether the mass at the origin is lighter or heavier than the others, there is a normal model with frequency below or above, respectively, the continuum frequency band.

Mathematically, the spectral phenomena can be understood by considering a staggering transformation $[5,6]$ on the one-particle quantum mechanical Hilbert space $\ell_{2}\left(\mathbb{Z}^{d-1}\right)$. The staggering transformation maps the attractive $\delta$ potential Hamiltonian operator to the repulsive one, up to a constant factor and an additive shift. In terms of eigenfunctions, the staggering transformation maps the nodeless bound state eigenfunction of the attractive case to an eigenfunction of maximum oscillation, i.e. it alternates signs for adjacent points on 
the lattice. This eigenfunction is the bound state eigenfunction above the band for the repulsive $\delta$ potential Hamiltonian.

In this paper, keeping the ladder approximation, we show the existence of similar spectral phenomena as described above for the case $N>1$. Extending the spectral results to the case where Gaussian domination does hold, our main result is that two distinct bound states exist above the two-particle band if $\alpha_{N}<0$.

In order to understand the role played by Gaussian domination at a more structural level, our presentation of the underlying spectral problem here differs from the previous ones in the sense that the ladder approximation is obtained in a clear and direct way which shows naturally how Gaussian domination comes into play in determining the existence or non-existence of two-particle bound states. We also make a correspondence between the B-S equation for these $\mathrm{O}(N)$ spin models and a two-particle lattice Schrödinger Hamiltonian operator with a spin-dependent delta potential, which elucidates the two-particle bound state structure below and above the two-particle band for $N>1$. Furthermore, we point out that, to prove our spectral results, we formulate a non-customary procedure for obtaining correlation functions (cf's) with the proper decay rates. Although these cf's are well-known for bosonic field theories as ours, we note that choosing proper cf's in more complex models is not a trivial matter, so that our formulation can be viewed as a more general guide.

Our spectral results can be extended beyond the ladder approximation to the complete model by adapting the methods of $[3,4,8]$. Also, it is important to stress that Gaussian domination inequalities (including also more general correlations) have been proven only for the abelian cases $N=1,2$ (see [9]), for any $\beta$. They are not known to hold in the non-abelian case $N \geq 3$, which we treat here for small $\beta$.

We now give a more detailed account of the correspondence between the $\mathrm{O}(N)$ classical spin systems and the quantum field models and introduce the associated e-m operators.

For $\beta \ll 1$, the infinite lattice $\mathrm{cf}$ 's $\langle\cdot\rangle_{\beta}$, are obtained by a polymer expansion, and are translation invariant and analytic in $\beta$. The connected (truncated) cf's have exponential tree decay, with a rate of at least $-(1-\epsilon) \ln (\beta)$, where $\epsilon \rightarrow 0$ as $\beta \rightarrow 0$ (see [10] and [11]).

Associated with each spin model, there is an imaginary discrete time lattice quantum field theory with a Hamiltonian (i.e. energy) and momentum operators associated with translations on a $d-1$ dimensional sublattice. The Hamiltonian is given by minus the logarithm of the corresponding transfer matrix (see [10] and [12]). An associated quantum field theory is constructed using standard methods furnishing a Hilbert space $\mathcal{H}$, commuting self-adjoint e-m operators $H \geqslant 0, P_{k}, k=1,2, . . d-1$, time zero field operators $\hat{s}_{i}(\vec{x})=e^{i \vec{P} \cdot \vec{x}} \hat{s}_{i} e^{-i \vec{P} \cdot \vec{x}}$ and vacuum vector $\Omega$. Vacuum expectation values of products of imaginary-time Heisenberg operators are related to cf's by the Feynman-Kac (F-K) formula, with $x_{i}=\left(t_{i}, \vec{x}_{i}\right)$ and $t_{1} \leqslant t_{2} \leqslant \ldots \leqslant t_{n}$,

$$
\left(\Omega, \hat{s}_{i_{1}} e^{-H\left(t_{2}-t_{1}\right)} e^{-i \vec{P}\left(\vec{x}_{2}-\vec{x}_{1}\right)} \hat{s}_{i_{2}} \ldots e^{-H\left(t_{n}-t_{n-1}\right)} e^{-i \vec{P}\left(\vec{x}_{n}-\vec{x}_{n-1}\right)} \hat{s}_{i_{n}} \Omega\right)_{\mathcal{H}}=\left\langle s_{i_{1}}\left(x_{1}\right) \ldots s_{i_{n}}\left(x_{n}\right)\right\rangle_{\beta} .
$$

The decay rates of cf's are related to the e-m spectrum and the low-lying e-m spectrum has a particle interpretation. One-particle states are generated by vectors of the form $\hat{s}_{i}(\vec{x}) \Omega$ and two-particle states by $\hat{s}_{i}(\vec{x}) \hat{s}_{j}(\vec{y}) \Omega$. The e-m spectral points are denoted by $(E, \vec{p}), E \geqslant 0, \vec{p} \in T_{d-1}$ (the torus $[-\pi,-\pi)^{d-1}$ ), the point $(E, \overrightarrow{0})$ is called mass and denoted by $m \equiv m(\beta, N)$.

The e-m spectrum associated with one-particle states consists of an isolated dispersion curve

$$
w(\vec{p})=-\ln \frac{\beta\left\langle\vec{s}^{2}\right\rangle}{N}-\frac{2\left\langle\vec{s}^{2}\right\rangle(d-1)}{N} \beta+\frac{2\left\langle\vec{s}^{2}\right\rangle}{N} \beta \sum_{j=1}^{d-1}\left(1-\cos p_{j}\right)+O\left(\beta^{2}\right),
$$

such that $w(\vec{p}) \geqslant w(\overrightarrow{0}) \equiv m$ (see [11] for the details) and

$$
m=-\ln \frac{\beta\left\langle\vec{s}^{2}\right\rangle}{N}-\frac{2\left\langle\vec{s}^{2}\right\rangle(d-1)}{N} \beta+O\left(\beta^{2}\right) .
$$

There is also a two-particle band. For fixed momentum $\vec{p}$, this band is given by the values of $w\left(\vec{p}_{1}\right)+w\left(\vec{p}_{2}\right)$, with $\vec{p}_{1}+\vec{p}_{2}=\vec{p}$ fixed. For $\vec{p}=0$ the two-particle band has width

$$
W=2[w(\vec{\pi})-w(\overrightarrow{0})]=8 \beta \frac{\left\langle\vec{s}^{2}\right\rangle^{2}}{N}(d-1)+O\left(\beta^{2}\right),
$$

with $\vec{\pi}=(\pi, \ldots, \pi)$, and extends from the 2-particle threshold $2 m$ to $2 m+W$. 
This paper is organized as follows. In section 2, we analyze the lattice ferromagnetic classical vector spin system for $N>1$. We obtain the spectral pattern, in the Gaussian domination and the Gaussian subjugation cases, using a ladder approximation. Also we show that, under certain conditions, the bound states are symmetrically located with respect of the two-particle band. In section 3, we show how the obtained spectral pattern can be understood by relating it to the spectrum of a lattice Schrodinger Hamiltonian with attractive and repulsive delta potentials and using staggering transformations.

\section{Ladder Approximation and Bound States}

In this section, we obtain the low-lying e-m spectrum for the lattice quantum field theory associated with the lattice classical ferromagnetic $\mathrm{O}(\mathrm{N})$ spin systems. We first give a non-customary procedure for obtaining appropriate cf's which are to be used to detect spectral points. In the case of a real bosonic field theories, as treated here, the proper two and four-point functions are well known, but in other theories, e.g. gauge or gauge-matter $[13,14]$, the choice of the appropriate cf's with desirable decay properties is not obvious at all. Thus, the procedure presented below can be used as a general guide for obtaining cf's in other more complex models.

As usual, it is enough to consider the inner product of Hilbert space $\mathcal{H}$ objects

$$
\left(\hat{\Phi}, \hat{T}_{0}^{\left|x_{0}\right|} \hat{\overrightarrow{\vec{T}} \vec{x}} \hat{\Psi}\right)_{\mathcal{H}}
$$

where $x=\left(x_{0}, \vec{x}\right) \in \mathbb{Z}^{d}, \hat{T}_{0}=e^{-H}, \vec{T} \vec{x}=e^{i \vec{P} \cdot \vec{x}}$ and $\hat{\Phi}, \hat{\Psi}$ belong to a dense subset of $\mathcal{H}$.

For $\hat{\Phi}$ and $\hat{\Psi}$ with finite support in $x_{0} \geqslant 0$, we have the Feyman-Kac formula (F-K) ([10]), for $x_{0} \geqslant 0$,

$$
\left(\hat{\Phi}, \hat{T}_{0}^{x_{0}} \hat{\overrightarrow{T^{x}}} \hat{\Psi}\right)_{\mathcal{H}}=\left\langle\left(T_{0}^{x_{0}} \vec{T}^{\vec{x}} \Psi\right) \Theta \Phi\right\rangle_{\beta},
$$

with Hilbert space vectors and operators in the left-hand-side and random variables in the right-hand-side. Also, $T_{0}$ is the time translation by one unit and $\vec{T}^{\vec{x}}$ is a space translation by $\vec{x}$. $\Theta$ is the standard reflection operator.

Denoting by $\chi(w)$ the translation of $\chi$ by $w$, and setting $x_{0}=v_{0}-u_{0}$, the F-K formula, for $\Phi$ and $\Psi$ supported on $x_{0}=0$, takes the form

$$
\left(\hat{\Phi}, \hat{T}_{0}^{\left|x_{0}\right| \hat{\vec{T}} \vec{x}} \hat{\Psi}\right)_{\mathcal{H}}= \begin{cases}\left\langle\left[\Theta \Phi\left(u_{0}, 0\right)\right] \Psi\left(v_{0}, \vec{x}\right)\right\rangle_{\beta}, & \text { if } u_{0}<v_{0} \\ \left\langle\Phi\left(u_{0}, 0\right) \Theta \Psi\left(v_{0}, \vec{x}\right)\right\rangle_{\beta}^{*}, & \text { if } u_{0}>v_{0}\end{cases}
$$

where $*$ stands for complex conjugation. The spectral representation of $\mathcal{G}_{\Phi, \Psi}(x)$ and $\tilde{\mathcal{G}}_{\Phi, \Psi}(p)$, the Fourier transform of $\mathcal{G}_{\Phi, \Psi}(x)$, can be obtained using the spectral representation

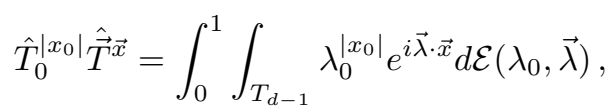

where $\mathcal{E}\left(\lambda_{0}, \vec{\lambda}\right)$ is the joint spectral family of $\hat{T}_{0}, \vec{P}$. Thus,

$$
\tilde{\mathcal{G}}_{\Phi, \Psi}(p)=(2 \pi)^{d-1} \int_{0}^{1} \int_{T_{d-1}}\left[\frac{1}{e^{i p_{0}}-\lambda_{0}}+\frac{1}{e^{-i p_{0}}-\lambda_{0}}\right] \delta(\vec{p}-\vec{\lambda}) d\left(\hat{\Phi}, \mathcal{E}\left(\lambda_{0}, \vec{\lambda}\right) \hat{\Psi}\right)_{\mathcal{H}},
$$

and, for $\vec{p}$ fixed, the singularities in the complex $p_{0}$ plane of $\tilde{\mathcal{G}}$ are points in the e-m spectrum.

We now consider the special cases of vectors $\Phi$ and $\Psi$, which corresponds to the usual two and four-point cf's, which are used to determine the low-lying e-m spectrum. The determination of the $p_{0}$ singularities of $\tilde{\mathcal{G}}_{\Phi, \Psi}(p)$, for $\vec{p}=0$, will be the method that we use to detect mass spectrum for the special cases of appropriate two and four-point functions.

Let $\Phi=s_{i_{1}}\left(0, \vec{x}_{1}\right)$ and $\Psi=s_{i_{2}}\left(0, \vec{x}_{2}\right)$. The corresponding $\mathcal{G}$ is, with $x=\left(v_{0}-u_{0}, \vec{x}\right)$,

$$
\mathcal{G}_{\vec{x}_{1}, i_{1}, \vec{x}_{2}, i_{2}}=\left\langle s_{i_{1}}\left(u_{0}, \vec{x}_{1}\right) s_{i_{2}}\left(v_{0}, \vec{x}_{2}+\vec{x}\right)\right\rangle_{\beta} .
$$


We define the usual two-point function by

$$
G_{i_{1}, i_{2}}(x)=\mathcal{G}_{\vec{x}_{1}, i_{1}, \vec{x}_{2}, i_{2}}\left(v_{0}-u_{0}, \overrightarrow{0}\right),
$$

where $\vec{x}=\vec{x}_{1}-\vec{x}_{2}$. The Fourier transforms are related by

$$
\tilde{G}_{i_{1} i_{2}}(p)=e^{i \vec{p} \cdot\left(\vec{x}_{1}-\vec{x}_{2}\right)} \tilde{\mathcal{G}}_{\vec{x}_{1}, i_{1}, \vec{x}_{2}, i_{2}}(p),
$$

so that $p_{0}$ singularities of $\tilde{G}_{i_{1} i_{2}}(p)$ and $\tilde{\mathcal{G}}(p)$ are the same.

Now, we take $\Phi=s_{i_{1}}\left(0, \vec{x}_{1}\right) s_{i_{2}}\left(0, \vec{x}_{2}\right)$ and $\Psi=s_{i_{3}}\left(0, \vec{x}_{3}\right) s_{i_{4}}\left(0, \vec{x}_{4}\right)$. The corresponding four-point cf is

$$
\mathcal{G}_{\vec{x}_{1}, i_{1}, \vec{x}_{2}, i_{2}, \vec{x}_{3}, i_{3}, \vec{x}_{4}, i_{4}}(x)=\left\langle s_{i_{1}}\left(v_{0}, \vec{x}_{1}\right) s_{i_{2}}\left(v_{0}, \vec{x}_{2}\right) s_{i_{3}}\left(u_{0}, \vec{x}_{3}+\vec{x}\right) s_{i_{4}}\left(u_{0}, \vec{x}_{4}+\vec{x}\right)\right\rangle_{\beta} .
$$

The usual four-point function is

$$
S_{i_{1} i_{2} i_{3} i_{4}}\left(x_{1}, x_{2} x_{3}, x_{4}\right)=\left\langle s_{i_{1}}\left(x_{1}\right) s_{i_{2}}\left(x_{2}\right) s_{i_{3}}\left(x_{3}\right) s_{i_{4}}\left(x_{4}\right)\right\rangle_{\beta}=S_{i_{1} i_{2} i_{3} i_{4}}\left(0, x_{2}-x_{1}, x_{3}-x_{1}, x_{4}-x_{1}\right),
$$

and, from now on, we use the equal time representation, where $x_{1}^{0}=x_{2}^{0}$ and $x_{3}^{0}=x_{4}^{0}$. In relative coordinates, $\vec{\xi}=\vec{x}_{2}-\vec{x}_{1}, \vec{\eta}=\vec{x}_{4}-\vec{x}_{3}$ and $\tau=x_{3}-x_{2}$, the usual four-point $\mathrm{cf}$ is

$$
S_{i_{1} i_{2} i_{3} i_{4}}^{\prime}(\vec{\xi}, \vec{\eta}, \tau)=S_{i_{1} i_{2} i_{3} i_{4}}\left(0, \vec{x}_{2}-\vec{x}_{1}=\vec{\xi}, x_{3}-\vec{x}_{1}=\vec{\xi}+\tau, x_{4}-\vec{x}_{1}=\vec{\xi}+\vec{\eta}+\tau\right),
$$

and we drop the prime from $S^{\prime}$. The Fourier transform of $S_{i_{1} i_{2} i_{3} i_{4}}(\vec{\xi}, \vec{\eta}, \tau)$ in the $\tau$ variable only is given by

$$
\hat{S}_{i_{1} i_{2} i_{3} i_{4}}(\vec{\xi}, \vec{\eta}, k),
$$

where $k=\left(k_{0}, \vec{k}\right)$. We have the relation

$$
\tilde{\mathcal{G}}_{\vec{x}_{1}, i_{1}, \vec{x}_{2}, i_{2}, \vec{x}_{3}, i_{3}, \vec{x}_{4}, i_{4}}(k)=e^{i \vec{k}\left(\vec{x}_{3}-\vec{x}_{2}\right)} \hat{S}_{i_{1} i_{2} i_{3} i_{4}}(\vec{\xi}, \vec{\eta}, k) .
$$

So, the $k_{0}$ singularities of $\tilde{\mathcal{G}}_{\vec{x}_{1}, i_{1}, \vec{x}_{2}, i_{2}, \vec{x}_{3}, i_{3}, \vec{x}_{4}, i_{4}}(k)$ and $\hat{S}_{i_{1} i_{2} i_{3} i_{4}}\left(\vec{\xi}, \vec{\eta}, k^{0}\right)$ are the same.

We now turn to the Bethe-Salpeter (B-S) equation which, in operator form, is

$$
D=D^{0}+D K D^{0},
$$

where $D, D^{0}$ and $K$ are taken as matrix operators acting in $s \ell_{2}(A)$, the symmetric subspace of $\ell_{2}(A)$, where $A=\left\{\left(x_{1}, i_{1}, x_{2}, i_{2}\right) \in\left(\mathbb{Z}^{d} \times S\right) \times\left(\mathbb{Z}^{d} \times S\right) \mid x_{1}^{0}=x_{2}^{0}\right\}$ and $S=1,2 \ldots, N$. Here, we are using the equal time representation for the B-S equation (see $[2,3]$ for more details).

We point out that there is a correspondence between this equation and a lattice Schrödinger resolvent equation for a non-relativistic two-body quantum mechanical Hamiltonian [6]. $D$ denotes the partially truncated four-point function with kernel given by

$$
D_{i_{1} i_{2} i_{3} i_{4}}\left(x_{1} x_{2} x_{3} x_{4}\right) \equiv\left\langle s_{i_{1}}\left(x_{1}\right) s_{i_{2}}\left(x_{2}\right) s_{i_{3}}\left(x_{3}\right) s_{i_{4}}\left(x_{4}\right)\right\rangle_{\beta}-\left\langle s_{i_{1}}\left(x_{1}\right) s_{i_{2}}\left(x_{2}\right)\right\rangle_{\beta}\left\langle s_{i_{3}}\left(x_{3}\right) s_{i_{4}}\left(x_{4}\right)\right\rangle_{\beta},
$$

and corresponds to the interacting resolvent. By the F-K formula, the subtraction removes vacuum (zero) spectral contributions and $D$ has temporal decay in $\tau_{0}$. The operator $D^{0}$, with kernel defined by

$$
D_{i_{1} i_{2} i_{3} i_{4}}^{0}\left(x_{1} x_{2} x_{3} x_{4}\right) \equiv\left\langle s_{i_{1}}\left(x_{1}\right) s_{i_{3}}\left(x_{3}\right)\right\rangle_{\beta}\left\langle s_{i_{2}}\left(x_{2}\right) s_{i_{4}}\left(x_{4}\right)\right\rangle_{\beta}+\left\langle s_{i_{1}}\left(x_{1}\right) s_{i_{4}}\left(x_{4}\right)\right\rangle_{\beta}\left\langle s_{i_{2}}\left(x_{2}\right) s_{i_{3}}\left(x_{3}\right)\right\rangle_{\beta},
$$

corresponds to the free Hamiltonian resolvent. Finally, the Bethe-Salpeter operator $K=D_{0}^{-1}-D^{-1}$ corresponds to minus the interacting potential.

We now give a simple derivation of our approximation to the B-S kernel, which we call the ladder approximation, and which turns out to be $\beta$ independent for our $\mathrm{O}(N)$ models. We use the fact that $D=D_{0}+D^{T}$, where $D^{T}$ is the connected (truncated) four-point function and that, at $\beta=0, D^{T}$ vanishes for non-coincident points. 
Analyzing only the $\beta$-independent contributions to this operator, we have, for any $j=1, \ldots, N$,

$$
\begin{aligned}
{\left[D_{\beta=0}^{0} f\right]_{i_{1} i_{2}}\left(x_{1}, x_{2}\right) } & =2\left\langle s_{j}^{2}\right\rangle^{2} f_{i_{1} i_{2}}\left(x_{1}, x_{2}\right) \\
{\left[D_{\beta=0} f\right]_{i_{1} i_{2}}\left(x_{1}, x_{2}\right) } & =\left[D_{\beta=0}^{0} f\right]_{i_{1} i_{2}}\left(x_{1}, x_{2}\right)+\left[D_{\beta=0}^{T} f\right]_{i_{1} i_{2}}\left(x_{1}, x_{2}\right) \\
& =2\left\langle s_{j}^{2}\right\rangle^{2} f_{i_{1} i_{2}}\left(x_{1}, x_{2}\right)+T_{i_{1} i_{2} i_{3} i_{4}} \delta\left(x_{1}-x_{2}\right) f_{i_{3} i_{4}}\left(x_{1}, x_{2}\right)
\end{aligned}
$$

where $T_{i_{1} i_{2} i_{3} i_{4}}=\left\langle s_{i_{1}} s_{i_{2}} s_{i_{3}} s_{i_{4}}\right\rangle-\left\langle s_{i_{1}} s_{i_{2}}\right\rangle\left\langle s_{i_{3}} s_{i_{4}}\right\rangle-\left\langle s_{i_{1}} s_{i_{3}}\right\rangle\left\langle s_{i_{2}} s_{i_{4}}\right\rangle-\left\langle s_{i_{1}} s_{i_{4}}\right\rangle\left\langle s_{i_{2}} s_{i_{3}}\right\rangle$. So, $D_{\beta=0}^{0}$ is a multiple of the identity, and $D_{\beta=0}$ is a multiplication operator in space-time variables, but not in the spin indices. Introducing the commuting orthogonal spin projections $([4])$

$$
\begin{aligned}
\mathcal{P}_{i_{1} i_{2} i_{3} i_{4}}^{0} & =\frac{\delta_{i_{1} i_{2}}}{N} \delta_{i_{3} i_{4}}, \\
\mathcal{P}_{i_{1} i_{2} i_{3} i_{4}}^{2} & =\frac{1}{2}\left[\delta_{i_{1} i_{3}} \delta_{i_{2} i_{4}}+\delta_{i_{1} i_{4}} \delta_{i_{2} i_{3}}-\frac{2}{N} \delta_{i_{1} i_{2}} \delta_{i_{3} i_{4}}\right],
\end{aligned}
$$

with eigenvalue one (symmetric) eigenvectors given by $w_{i_{1} i_{2}}^{(0)}=\delta_{i_{1} i_{2}}$, for $\mathcal{P}^{0}$, and $w_{i_{1} i_{2}}^{(r)}=\delta_{i_{1} 1} \delta_{i_{2} 1}-\delta_{i_{1} r} \delta_{i_{2} r}$, $r=2, \ldots, N$, and $w_{i_{1} i_{2}}^{(i j)}=\delta_{i_{1} i} \delta_{i_{2} j}+\delta_{i_{1} j} \delta_{i_{2} i}, 1 \leq i<j \leq N$, for $\mathcal{P}^{2}$; by direct computation, we see that $T$ commutes with $\mathcal{P}^{0}$ and $\mathcal{P}^{2}$. Also, we obtain $\mathcal{P}^{0} T=T \mathcal{P}^{0}=\mu_{0} \mathcal{P}^{0}$ and $\mathcal{P}^{2} T=T \mathcal{P}^{2}=\mu_{2} \mathcal{P}^{2}$. We have the properties

$$
T=\mu_{0} \mathcal{P}^{0}+\mu_{2} \mathcal{P}^{2} \quad, \quad I=\mathcal{P}^{0}+\mathcal{P}^{2}
$$

where, using $\alpha_{N}$ defined in Eq. (2),

$$
\begin{aligned}
\mu_{0} & =\sum_{k}\left[\left\langle s_{k}^{2} s_{3}^{2}\right\rangle-\left\langle s_{k}^{2}\right\rangle\left\langle s_{3}^{2}\right\rangle-2\left\langle s_{k} s_{3}\right\rangle^{2}\right] \\
& =\frac{1}{N}\left[\left\langle(\vec{s} \cdot \vec{s})^{2}\right\rangle-\frac{N+2}{N}\langle\vec{s} \cdot \vec{s}\rangle^{2}\right]=\frac{\alpha_{N}}{N} \\
\mu_{2} & =2\left[\left\langle s_{1}^{2} s_{2}^{2}\right\rangle-\left\langle s_{1}^{2}\right\rangle^{2}\right]=\frac{2}{N(N+2)}\left\langle(\vec{s} \cdot \vec{s})^{2}\right\rangle-\frac{N+2}{N}\langle\vec{s} \cdot \vec{s}\rangle^{2}=2 \frac{\alpha_{N}}{N(N+2)}
\end{aligned}
$$

where we use $\mathrm{O}(N)$ invariance to get the relations $\left\langle s_{1}^{2}\right\rangle=\left\langle\vec{s}^{2}\right\rangle / N$ and $\left\langle s_{1}^{2} s_{2}^{2}\right\rangle=\left\langle\vec{s}^{4}\right\rangle /[N(N+2)]=\left\langle\vec{s}_{1}^{4}\right\rangle / 3$.

Thus, we have

$$
K f=\frac{1}{2\left\langle s_{j}^{2}\right\rangle^{2}}\left[\mathcal{P}^{0}+\mathcal{P}^{2}\right] f-\frac{1}{2\left\langle s_{j}^{2}\right\rangle^{2}+\mu_{0} \delta} \mathcal{P}^{0} f-\frac{1}{2\left\langle s_{j}^{2}\right\rangle^{2}+\mu_{2} \delta} \mathcal{P}^{2} f+O(\beta),
$$

with $[\delta f]_{s_{1} s_{2}}\left(x_{1}, x_{2}\right)=\delta\left(x_{1}-x_{2}\right) f_{s_{1} s_{2}}\left(x_{1}, x_{2}\right)$. From this, we obtain,

$$
\begin{aligned}
& {\left[K \mathcal{P}^{0} f\right]_{i j}\left(x_{1}, x_{2}\right)=\lambda_{0} \delta\left(x_{1}-x_{2}\right)\left[\mathcal{P}^{0} f\right]_{i j}\left(x_{1}, x_{1}\right)+O(\beta),} \\
& {\left[K \mathcal{P}^{2} f\right]_{i j}\left(x_{1}, x_{2}\right)=\lambda_{2} \delta\left(x_{1}-x_{2}\right)\left[\mathcal{P}^{2} f\right]_{i j}\left(x_{1}, x_{1}\right)+O(\beta),}
\end{aligned}
$$

where

$$
\lambda_{0}=\frac{\alpha_{N} N^{2}}{2\left\langle\vec{s}^{2}\right\rangle^{2}} \frac{1}{\left\langle\vec{s}^{4}\right\rangle-\left\langle\vec{s}^{2}\right\rangle^{2}} ; \lambda_{2}=\frac{\alpha_{N} N^{2}}{2\left\langle\vec{s}^{2}\right\rangle^{2}} \frac{1}{\left\langle\vec{s}^{4}\right\rangle},
$$

and where we note that the Cauchy-Schwartz inequality ensures that the factor $\left\langle\vec{s}^{4}\right\rangle-\left\langle\vec{s}^{2}\right\rangle^{2}$ in the denominator is nonnegative. Thus, $\lambda_{0,2}$ and $\alpha_{N}$ have the same sign.

Equation (10) shows that $\lambda_{0}$ and $\lambda_{2}$ are eigenvalues of $K$, up to $O(\beta)$, with multiplicities one and $N(N+$ 1) $/ 2-1$, respectively (see [4] for an alternative brute force derivation). We also point out that $\mathcal{P}^{0}$ and $\mathcal{P}^{2}$ are projections on the subspaces (of rank two tensors) of irreducible representations of $\mathrm{O}(N)$.

In relative coordinates, the B-S equation reads

$$
\begin{aligned}
D_{i_{1} i_{2} i_{3} i_{4}}(\vec{\xi}, \vec{\eta}, \tau)=D_{i_{1} i_{2} i_{3} i_{4}}^{0}(\vec{\xi}, \vec{\eta}, \tau)+\int D_{i_{1} i_{2} j_{1} j_{2}}^{0}\left(\vec{\xi}, \vec{\xi}^{\prime}, \tau^{\prime}\right) K_{j_{1} j_{2} j_{3} j_{4}}\left(\vec{\xi}^{\prime}, \vec{\eta}^{\prime}, \tau-\tau^{\prime}-\tau^{\prime \prime}-\vec{\xi}^{\prime}-\vec{\eta}^{\prime}\right) \\
\times D_{j_{3} j_{4} i_{3} i_{4}}\left(\vec{\eta}^{\prime}, \vec{\eta}, \tau^{\prime \prime}\right) d \tau^{\prime} d \tau^{\prime \prime} d \vec{\xi}^{\prime} d \vec{\eta}^{\prime}
\end{aligned}
$$

where the sign $\int$ includes summation over the $j$ indices. 
From the basic exchange symmetry properties $x_{1} i_{1} \rightleftarrows x_{2} i_{2}$ and $x_{3} i_{3} \rightleftarrows x_{4} i_{4}$, we obtain the relations

$$
\begin{aligned}
& D_{i_{1} i_{2} i_{3} i_{4}}(\vec{\xi}, \vec{\eta}, \tau)=D_{i_{2} i_{1} i_{3} i_{4}}(-\vec{\xi}, \vec{\eta}, \tau+\vec{\xi}), \\
& D_{i_{1} i_{2} i_{3} i_{4}}(\vec{\xi}, \vec{\eta}, \tau)=D_{i_{1} i_{2} i_{4} i_{3}}(\vec{\xi},-\vec{\eta}, \tau+\vec{\eta}),
\end{aligned}
$$

so that for the Fourier transform in $\tau$, with $k_{0} \equiv\left(k_{0}, \vec{k}=\overrightarrow{0}\right), \hat{D}_{i_{1} i_{2} i_{3} i_{4}}\left(\vec{\xi}, \vec{\eta}, k_{0}\right)=\hat{D}_{i_{2} i_{1} i_{3} i_{4}}\left(-\vec{\xi}, \vec{\eta}, k_{0}\right)$ and $\hat{D}_{i_{1} i_{2} i_{3} i_{4}}\left(\vec{\xi}, \vec{\eta}, k_{0}\right)=\hat{D}_{i_{1} i_{2} i_{4} i_{3}}\left(\vec{\xi},-\vec{\eta}, k_{0}\right)$, and similarly for $D_{0}, K$ and $\hat{D}_{0}, \hat{K}$. $\hat{D}_{i_{1} i_{2} i_{3} i_{4}}\left(\vec{\xi}, \vec{\eta}, k_{0}\right)$, etc, act on functions $f_{i_{1} i_{2}}(\vec{\xi})$, obeying the property $f_{i_{1} i_{2}}(\vec{\xi})=f_{i_{2} i_{1}}(-\vec{\xi})$. Thus for $k=k_{0}$, the B-S equation becomes

$$
\begin{array}{r}
\hat{D}_{i_{1} i_{2} i_{3} i_{4}}\left(\vec{\xi}, \vec{\eta}, k_{0}\right)=\hat{D}_{i_{1} i_{2} i_{3} i_{4}}^{0}\left(\vec{\xi}, \vec{\eta}, k_{0}\right)+\int \hat{D}_{i_{1} i_{2} j_{1} j_{2}}^{0}\left(\vec{\xi}, \vec{\xi}^{\prime}, k_{0}\right) \hat{K}_{j_{1} j_{2} j_{3} j_{4}}\left(\vec{\xi}^{\prime}, \vec{\eta}^{\prime}, k_{0}\right) \\
\times \hat{D}_{j_{3} j_{4} i_{3} i_{4}}\left(\vec{\eta}^{\prime}, \vec{\eta}, k_{0}\right) d \vec{\xi}^{\prime} d \vec{\eta}^{\prime} .
\end{array}
$$

At this point, we notice that the original B-S equation in the $x_{i}$ coordinates, which corresponds to a two-particle lattice Schrödinger equation, since $\left(k_{0}, \vec{k}\right)=\left(k_{0}, \overrightarrow{0}\right)$ is fixed, now corresponds to a one particle lattice Schrödinger resolvent equation, with zero system momentum and external potential $-\hat{K}$. $k_{0}$ acts as a spectral parameter.

Next, we determine the solutions to Eq. (12) in the ladder approximation. Call $L$ the $\beta$-independent approximation to $K$, with kernel $L(\vec{\xi}, \vec{\eta}, \tau)=\delta(\vec{\xi}) \delta(\vec{\eta}) \delta(\tau) \ell$, where

$$
\ell=\lambda_{0} \mathcal{P}^{0}+\lambda_{2} \mathcal{P}^{2} .
$$

In this approximation, suppressing the $k_{0}=\left(k_{0}, \overrightarrow{0}\right)$ dependence, the B-S equation (12) is

$$
\hat{D}(\vec{\xi}, \vec{\eta})=\hat{D}^{0}(\vec{\xi}, \vec{\eta})+\hat{D}^{0}(\vec{\xi}, \overrightarrow{0}) \ell \hat{D}(\overrightarrow{0}, \vec{\eta}),
$$

with solution

$$
\hat{D}(\vec{\xi}, \vec{\eta})=\hat{D}^{0}(\vec{\xi}, \vec{\eta})+\hat{D}^{0}(\vec{\xi}, \overrightarrow{0})\left[1-\ell \hat{D}^{0}(\overrightarrow{0}, \overrightarrow{0})\right]^{-1} \hat{D}^{0}(\overrightarrow{0}, \vec{\eta})
$$

where

$$
\begin{aligned}
\hat{D}_{i_{1} i_{2} i_{3} i_{4}}^{0}(\overrightarrow{0}, \overrightarrow{0}) & =\int\left[\left\langle s_{i_{1}}(0) s_{i_{3}}(\tau)\right\rangle_{\beta}\left\langle s_{i_{2}}(0) s_{i_{4}}(\tau)\right\rangle_{\beta}+\left\langle s_{i_{1}}(0) s_{i_{4}}(\tau)\right\rangle_{\beta}\left\langle s_{i_{2}}(0) s_{i_{3}}(\tau)\right\rangle_{\beta}\right] e^{-i k_{0} \tau_{0}} d \tau_{0} d \vec{\tau} \\
& =\left(\delta_{i_{1} i_{3}} \delta_{i_{2} i_{4}}+\delta_{i_{1} i_{4}} \delta_{i_{2} i_{3}}\right) \int\left\langle s_{1}(0) s_{1}(\tau)\right\rangle_{\beta}^{2} e^{-i k_{0} \tau_{0}} d \tau_{0} d \vec{\tau} \\
& \equiv Q_{i_{1} i_{2} i_{3} i_{4}} R\left(k_{0}\right),
\end{aligned}
$$

where

$$
R\left(k_{0}\right)=\int\left\langle s_{1}(0) s_{1}(\tau)\right\rangle_{\beta}^{2} e^{-i k_{0} \tau_{0}} d \tau_{0} d \vec{\tau} .
$$

Q commutes with $\mathcal{P}^{0}$ and $\mathcal{P}^{2}$ so $\ell D_{0}(\overrightarrow{0}, \overrightarrow{0})$ has eigenvalues $2 \lambda_{0} R\left(k_{0}\right)$ and $2 \lambda_{2} R\left(k_{0}\right)$, with multiplicities one and $-1+N(N+1) / 2$, respectively.

$R\left(k_{0}\right)$ can be obtained from the spectral representation of the two-point $\mathrm{cf}$ (see [12] and [11] for the details). We have,

$$
\left\langle s_{1}(0) s_{1}(x)\right\rangle_{\beta}=\int_{0}^{\infty} \int_{T_{d-1}} e^{i \vec{p} \cdot \vec{x}-E\left|x_{0}\right|} d \sigma_{\vec{p}}(E) d \vec{p} \equiv G_{11}(x),
$$

where, for $w(\vec{p})$ given in Eq. (4),

$$
d \sigma_{\vec{p}}(E)=Z(\vec{p}, \beta) \delta(E-\omega(\vec{p})) d E+d \hat{\sigma}_{\vec{p}}(E)
$$

and $Z(\vec{p}, \beta)=\left.\frac{\partial \tilde{\Gamma}}{\partial \chi}\left(p_{0}=i \chi, \vec{p}\right)\right|_{\chi=\omega(\vec{p})}$, where $\tilde{\Gamma}(p)=\left[\tilde{G}_{11}(p)\right]^{-1}$. Both $d \sigma_{\vec{p}}(E)$ and $d \hat{\sigma}_{\vec{p}}(E)$ are positive measures. $d \hat{\sigma}_{\vec{p}}(E)$ has support in $(\bar{m}, \infty), \bar{m} \approx-3 \ln \beta$ is a lower bound for the onset of three-particle spectrum. The decomposition of $d \sigma_{\vec{p}}(E) d \vec{p}$ gives a separation into one-particle and three or more particle contributions (related to the $\left.d \hat{\sigma}_{\vec{p}}(E)\right)$. Hence, $Z(\vec{p}, \beta)$ is positive for any $\vec{p}$ and real $\beta>0$.

Using this representation, and taking in account only the product of one-particle contribution (associated with $Z(\vec{p}, \beta) \delta(E-\omega(\vec{p})) d E$ ), we obtain (see [2] and [3])

$$
R\left(k_{0}\right)=2(2 \pi)^{d-1} \int_{T_{d-1}} \frac{\sinh (2 \omega(\vec{p}))[Z(\vec{p}, \beta)]^{2}}{\cosh (2 \omega(\vec{p}))-\cos \left(k_{0}\right)} d \vec{p}+O(\beta)
$$


where $Z(\vec{p}, \beta)=(2 \pi)^{1-d}\left\langle\vec{s}^{2}\right\rangle / N+O(\beta)$.

From this representation, we see that $R\left(k_{0}\right)$ is analytic outside the band. A more general spectral representation for $\hat{D}^{0}(\vec{\xi}, \vec{\eta})$ (see [2]) also shows that it is analytic on the imaginary $k_{0}$ axis outside the band and for $\left|\mathcal{I} \mathrm{m} \mathrm{m} k_{0}\right|<3 \mathrm{~m}$. Hence, the singularities of Eq. (14) are the zeroes of $\operatorname{det}\left[1-\ell \hat{D}^{0}(\overrightarrow{0}, \overrightarrow{0})\right]$. Also, taking $k_{0}=i\left(2 m+\frac{W}{2}+\chi\right)$, for $W$ given in Eq. (6), an inspection of the above representation for $R\left(k_{0}\right)$ shows that $R\left(k_{0}\right)$ is positive below the band, i.e. for $\chi<-\frac{W}{2}$, and negative $k_{0}$ above the band, i.e. for $\chi>\frac{W}{2}$. of

The $k_{0}$ singularities of $\hat{D}\left(\vec{\xi}, \vec{\eta}, k_{0}\right)$ in Eq. (14), for $\mathcal{I} \mathrm{m} \mathrm{m}\left(k_{0}\right) \in(0,2 m) \cup(2 m+W, 3 m)$, come from solutions

$$
2 \lambda_{i} \sum_{\tau}\left\langle s_{1}(0) s_{1}(\tau)\right\rangle^{2} e^{2 m \tau_{0}} e^{\left(\frac{W}{2}+\chi\right) \tau_{0}}=1
$$

We give an intuitive argument for the bound state formula based on the behavior of $\left\langle s_{1}(0) s_{1}(\tau)\right\rangle_{\beta}$. A rigorous argument using the convolution form in momentum space of the above condition and the spectral representation of two-point functions can be found in reference [4]. To rigourously control all contributions, a polymer expansion can be used. Expanding $\left\langle s_{1}(0) s_{1}(\tau)\right\rangle_{\beta}$ to leading order in $\beta$ we obtain $\left\langle s_{1}(0) s_{1}(\tau)\right\rangle_{\beta} \approx \beta^{\left|\tau_{0}\right|+|\vec{\tau}|}\left\langle s_{1}^{2}\right\rangle\left|\tau_{0}\right|+|\vec{\tau}|+1$. Also, using $m(\beta)$ from Eq. (5), we obtain the bound state condition to the leading order in $\beta, j=0,2$,

$$
2\left\langle s_{1}^{2}\right\rangle \lambda_{j}\left(1-e^{\frac{W}{2}+\chi}\right)=1 .
$$

From condition (16), we see that there is a bound state solution above (below) the band for negative (positive) $\lambda_{j}$, and we recall that, from Eq. (11), the sign of $\lambda_{j}$ is the same as the sign of $\alpha_{N}$.

Let $\varepsilon_{j}^{-}=-\chi-W / 2\left(\varepsilon_{j}^{+}=\chi-W / 2\right)$ denote the binding energy for the attractive (repulsive) case. From Eq. (16), we find

$$
\begin{aligned}
e^{-\varepsilon_{j}^{-}} & =\left(\delta_{2 j} \frac{\langle\vec{s} \cdot \vec{s}\rangle_{-}}{\left\langle(\vec{s} \cdot \vec{s})^{2}\right\rangle_{-}}+\frac{2}{N} \frac{\langle\vec{s} \cdot \vec{s}\rangle_{-}^{2}}{\left\langle(\vec{s} \cdot \vec{s})^{2}\right\rangle_{-}-\delta_{0 j}\langle\vec{s} \cdot \vec{s}\rangle_{-}}\right), \\
e^{+\varepsilon_{j}^{+}} & =\left(\delta_{2 j} \frac{\langle\vec{s} \cdot \vec{s}\rangle_{+}}{\left\langle(\vec{s} \cdot \vec{s})^{2}\right\rangle_{+}}+\frac{2}{N} \frac{\langle\vec{s} \cdot \vec{s}\rangle_{+}^{2}}{\left\langle(\vec{s} \cdot \vec{s})^{2}\right\rangle_{+}-\delta_{0 j}\langle\vec{s} \cdot \vec{s}\rangle_{+}}\right) e^{-W} ;
\end{aligned}
$$

where $\langle\cdot\rangle_{-}\left(\langle\cdot\rangle_{+}\right)$means the ssd expectation for the attractive (repulsive) case.

Now we turn to the analysis of the relation between the binding energies for these two cases, and show that, under certain conditions, the binding energies are the same, i.e. $\varepsilon_{j}^{-}=\varepsilon_{j}^{+}$.

If the bands are the same in both cases, by Eq. (6), we must have $\langle\vec{s} \cdot \vec{s}\rangle_{-}^{2}=\langle\vec{s} \cdot \vec{s}\rangle_{+}^{2}$. Also, taking the corresponding $\lambda_{j}$ for the attractive to be minus the $\lambda_{j}$ for repulsive case, we have

$$
\left\langle\vec{s}^{4}\right\rangle_{-}\left\langle\vec{s}^{4}\right\rangle_{+}=\left[\frac{N+2}{N}\right]^{2}\langle\vec{s} \cdot \vec{s}\rangle^{2} .
$$

So by a direct calculation, we show that $\varepsilon_{j}^{-}=\varepsilon_{j}^{+}$. We show that, with a very weak restriction, the attractive and repulsive cases have the same binding energies.

Thus, we have shown that for sufficiently high temperatures and in the ladder approximation that there are precisely two bound states below or above the band depending on the sign of the parameter $\alpha_{N}$, which characterizes the attractive $\left(\alpha_{N}>0\right.$ and bound states below the band) and the repulsive cases $\left(\alpha_{N}<0\right.$ and bound states above the band). We remark that $\alpha_{N}$ measures the gaussian domination. These bound states are symmetrically located with respect to the two particle band, when the ssd is such that relation (17) is verified.

In closing the section, we stress that if $\alpha_{N}=0$ no bound state occurs in $(0,2 m) \cup(2 m+W, 3 m)$.

\section{Staggering Transformations and Bound States}

As seen before, there is a correspondence between the B-S equation in the $x_{i}$ coordinate space and the Schrödinger resolvent equation for a two-particle quantum Hamiltonian. Hence, some of the spectral properties can be understood by looking at the spectrum for this two-particle Hamiltonian. In the ladder approximation, the corresponding potential is local, and is given by a spin dependent delta potential at the origin.

For this, consider the Hilbert space $\mathcal{H}$ given by the symmetric subspace of the space $\ell^{2}(B)$, where $B=$ $\left\{\left(\vec{x}_{1} i_{1}, \vec{x}_{2} i_{2}\right) \in\left(\mathbb{Z}^{d-1} \times S\right) \otimes\left(\mathbb{Z}^{d-1} \otimes S\right)\right\}$ and $S=\{1,2, \ldots, N\} ;$ and we write an element $f$ of $\mathcal{H}$ as $f_{i_{1} i_{2}}\left(\vec{x}_{1}, \vec{x}_{2}\right)$ 
$=f_{i_{2} i_{1}}\left(\vec{x}_{2}, \vec{x}_{1}\right)$; and a non-relativistic quantum Hamiltonian operator $H$ acting in $\mathcal{H}$ given by, with $\nu_{0}, \nu_{2} \in \mathbb{R}$,

$$
H=\left[\frac{-\Delta_{1}}{2 m}+\frac{-\Delta_{2}}{2 m}\right]+\left(\nu_{0} \mathcal{P}^{0}+\nu_{2} \mathcal{P}^{2}\right) \delta\left(x_{1}-x_{2}\right) \equiv H_{0}+\ell V,
$$

where $\Delta_{n=1,2}$ is the $\mathbb{Z}^{d-1}$ lattice Laplacian acting only on the $n$th lattice coordinate and $\mathcal{P}^{0}$ and $\mathcal{P}^{2}$ act only on the spin indices, and are respectively the spin zero and spin two tensor projections defined in Eqs. (8) and (9). For a function $g(\vec{x}), \vec{x} \in \mathbb{Z}^{d-1}$, we have $-\Delta g(\vec{x})=2(d-1) g(\vec{x})-\sum_{\epsilon= \pm 1} \sum_{j=1}^{d-1} g\left(\vec{x}+\epsilon e_{j}\right)$, for $e_{j}$ denoting the $j$ th lattice unit vector.

To make contact with the above spin models, we recall that in relative coordinates and for system momentum zero, the $\hat{D}_{0}$ term of the B-S equation corresponds to the resolvent of a free lattice Schrödinger operator $-c \beta \Delta$, where $c>0$ is a constant. Thus, taking $\beta$ small means a sufficiently large mass $m$ in the Schrödinger Hamiltonian of Eq. (18). We stress that we only analyze the equal mass case, since in our bosonic models the particles are identical and this is what occurs in the B-S equation.

We define the staggering transformation by

$$
U f_{i j}\left(\vec{x}_{1}, \vec{x}_{2}\right)=(-1)^{\sum_{i=1}^{d-1}\left(x_{1}^{i}+x_{2}^{i}\right)} f_{i j}\left(\vec{x}_{1}, \vec{x}_{2}\right), \quad f \in \mathcal{H} .
$$

By direct calculation, $U$ has the following properties

$$
U^{2}=I, U H=\left\{[4(d-1)] / m-\left(H_{0}+\ell \delta\right)\right\} U, \quad[\tilde{U f}]_{i j}\left(\vec{p}_{1}, \vec{p}_{2}\right)=\tilde{f}_{i j}\left(\vec{\pi}-\vec{p}_{1}, \vec{\pi}-\vec{p}_{2}\right),
$$

where here we set $\ell=\nu_{0} \mathcal{P}^{0}+\nu_{2} \mathcal{P}^{2}$. Equation (20) shows us that if we know the spectrum and eigenfunctions, for the attractive case, by the staggering transformation we obtain the spectrum and the eigenfunctions for the repulsive case, and vice-versa.

We now determine the spectrum of the Hamiltonian $H$ of Eq. (18). For this, we first consider the system self-adjoint momentum operator $\vec{P}$. Let $T(\vec{a})$ be the system translation operator by $\vec{a} \in \mathbb{Z}^{d-1}$ defined by $(T(\vec{a}) f)_{i j}\left(\vec{x}_{1}, \vec{x}_{2}\right)=f_{i j}\left(\vec{x}_{1}+\vec{a}, \vec{x}_{2}+\vec{a}\right) . T(\vec{a})$ is unitary so we write $T(\vec{a})=e^{i \vec{P} \cdot \vec{a}}$, which defines the self-adjoint $\vec{P}$. The spectrum of $\vec{P}$ is contained in $\mathbf{T}_{d-1}=(-\pi, \pi]^{d-1}$ and $\vec{P}$ commutes with $H$ and $H_{0}$.

We determine eigenfunctions of $H$. Using the eigenvalue one eigenvectors of the orthogonal projections $\mathcal{P}^{0}$ and $\mathcal{P}^{2}$ introduced in Eqs. (8) and (9), we write the eigenfunctions of $H$ in terms of an expansion in product of plane waves of the form

$$
\psi_{i_{1} i_{2}}^{(n)}\left(\vec{x}_{1}, \vec{x}_{2}, \vec{p}, \vec{k}=2 \vec{q}\right)=v_{i_{1} i_{2}}^{(n)} e^{i \vec{p} \cdot\left(\vec{x}_{1}-\vec{x}_{2}\right)} e^{i \vec{q} \cdot\left(\vec{x}_{1}+\vec{x}_{2}\right)},
$$

where $v_{i_{1} i_{2}}^{(n)} \equiv v^{(n)}, n=0,2$ is an eigenvector of $\mathcal{P}^{n}$, and $\vec{k}$ is the system momentum. Also, $\psi_{i j}^{(n)}\left(\vec{x}_{1}, \vec{x}_{2}, \vec{p}, \vec{k}\right)$ is an eigenfunction of the free Hamiltonian $H_{0}$, with eigenvalue

$$
\mathcal{T}(\vec{p}, \vec{q})=\frac{-\tilde{\Delta}(\vec{p}+\vec{q})}{2 m}+\frac{-\tilde{\Delta}(\vec{p}-\vec{q})}{2 m}=\frac{2}{m} \sum_{j=1}^{d-1}\left[1-\cos \left(p_{j}\right) \cos \left(k_{j}\right)\right] .
$$

We see that the eigenvalue does not split into a sum of center of mass and relative kinetic energy as in the continuum using center of mass and relative coordinates. However, we observe that $H_{0}$ is still a multiplication operator, and it has a band spectrum for any $d$, with a finite width which becomes zero if the system momentum $\vec{k}$ is equal to $\vec{\pi} \equiv(\pi, \ldots, \pi)$.

Consider the case of system momentum $\vec{k}=\overrightarrow{0}$. Here, we still have a band of continuum spectra in the interval $\left[0, \frac{4(d-1)}{m}\right]$. Next, we write the $H$ eigenfunctions as the superposition

$$
\phi^{(n)}\left(\vec{x}_{1}, \vec{x}_{2}\right)=\int_{T_{d-1}} v^{(n)} \tilde{b}^{(n)}(\vec{p}) e^{i \vec{p}\left(\vec{x}_{1}-\vec{x}_{2}\right)} d \vec{p},
$$

where $\tilde{b}^{(n)}(\vec{p})=\tilde{b}^{(n)}(-\vec{p})$. With this, the eigenvalue equation becomes, with $t(\vec{p})=\frac{1}{m} \sum_{j=1}^{d-1} 2\left(1-\cos p_{j}\right)$,

$$
\left(H-E_{n}\right) \phi^{(n)}\left(\vec{x}_{1}, \vec{x}_{2}\right)=v^{(n)} \int_{T_{d-1}}\left[t(\vec{p})++\nu_{n} \delta\left(\vec{x}_{1}-\vec{x}_{2}\right)-E_{n}\right] \tilde{b}^{(n)}(\vec{p}) e^{i \vec{p}\left(\vec{x}_{1}-\vec{x}_{2}\right)} d \vec{p} .
$$


Taking the Fourier transform in the $\vec{x}_{1}-\vec{x}_{2}$ variable gives

$$
\left[t(\vec{p})-E_{n}\right] \tilde{b}^{(n)}(\vec{p})+\nu_{n} \int_{T_{d-1}} \tilde{b}^{(n)}(\vec{p}) d \vec{p}=0 .
$$

Let us take $E_{n}<0$, which corresponds to energies below the continuous band. Dividing Eq. (22) by $\left[t(\vec{p})-E_{n}\right]$ and integrating gives, for $E_{n}<0$, which corresponds to energies below the continuous band, the bound state equation

$$
1+\nu_{n} \int_{T_{d-1}} \frac{1}{t(\vec{p})-E_{n}} d \vec{p}=0 .
$$

If a solution to Eq. (23) exists, then $\tilde{b}^{(n)}(\vec{p})$ is given by

$$
\tilde{b}^{(n)}(\vec{p})=\frac{-\nu_{n}}{t(\vec{p})-E_{n}} \int_{T_{d-1}} \tilde{b}^{(n)}\left(\vec{p}^{\prime}\right) d \vec{p}^{\prime}=\frac{c}{t(\vec{p})-E_{n}},
$$

where $c$ is a constant so that $\tilde{b}^{(n)}(\vec{p})=\tilde{b}^{(n)}(-\vec{p})$ as required.

Since $-\tilde{\Delta}(\vec{p}) \approx|\vec{p}|^{2}$, for sufficiently small $|\vec{p}|$, there are bound states $E_{n}, n=0,2$ with multiplicities one and $\frac{N(N+1)}{2}-1$, respectively, for $d-1=1,2$ and any $\nu_{n}<0$. For $d>3$, the integral in Eq. (23) converges for $E_{n}=0$, then there are critical values $\nu_{n}^{c} \equiv \nu_{n}^{c}(d)<0$ for a bound state to exist (see the Birman-Schwinger bounds in reference [15]). Also, applying Perron-Frobenius theorem to $e^{-H}$ (see [15]), the associated ground state eigenfunction $\phi^{(n)}\left(\vec{x}_{1}, \vec{x}_{2}\right)$ is positive, i.e $\phi^{(n)}\left(\vec{x}_{1}, \vec{x}_{2}\right)>0$ for any $n, \vec{x}_{1}$ and $\vec{x}_{2}$. Last, by the Payley-Wiener theorem [16], the strip analyticity of $\tilde{b}^{(n)}(\vec{p})$ of Eq. (24) implies exponential decay of the bound state wave function. This completes the description of $H$, for $\nu_{n}<0$.

Now using the intertwining property of Eq. (20), the spectrum of $H$ for $\nu_{n}>0$ consists of $\bigcup_{n}\left(\frac{4(d-1)}{m}+E_{n}\right) \cup$ $\left[0, \frac{4(d-1)}{m}\right]$. The surprising feature is the existence of the same spectral pattern of the attractive case, above the continuous spectrum. Besides, the value of the binding energy in the attractive and repulsive cases depend on the value of $\left|\nu_{n}\right|$. As the eigenfunction for the attractive case is positive, for the repulsive case the bound state eigenfunction has maximum oscillation by Eq. (19).

It is important to observe that the relationship between the spectra for the attractive and repulsive cases, as seen with help the of staggering transformations is exact for the $\mathrm{O}(N)$ spin systems, in the ladder approximation. The condition given by Eq. (17) and the requirement of uniqueness of the band in both cases, attractive and repulsive, are naturally incorporated in the context of staggering transformations.

\section{Concluding Remarks}

It is important to stress that in $[2,3]$ the spectral results for $N=1$ were obtained for the complete model, providing an extension beyond the ladder approximation, and that the same procedure also applies to extend the spectral results from [4] and ours, i.e. for the $N>1$ case, to the full model. Whether or not the degeneracies are preserved or broken when the full model is considered is an important open question. Also, there may appear bound states associated with anti-symmetric spin states in a higher order in $\beta$. We observe that, in our $O(\beta)$ analysis, only overall symmetric spin-space bound states occur. The anti-symmetric spin states which also have an anti-symmetric space part do not feel the delta function potential.

Moreover, we remark that the argument developed here to obtain the cf's with proper decay rates can also be applied to more complex models, they provide a general guide to obtain these $\mathrm{cf}$ 's. Besides, the procedure used to obtain the ladder approximation, clarifies the role played by Gaussian domination involving the truncated four-point function, as it defines the truncated $D$, that enters in the Bethe-Salpeter equation. In fact, for $\beta=0$, the connected four-point cf's only allow contributions for coincident points, and contain all information about Gaussian domination.

\section{Acknowledgments}

This work was partially supported by Pronex and CNPq. P.H.R. dos Anjos also acknowledges a scholarship from FAPESP. 


\section{References}

[1] R. Schor and Michael O'Carroll, Phys. Rev. E62, 1-5 (2000).

[2] R. Schor and Michael O'Carroll, J. Stat. Phys. 99, Nos. 5/6, 1207-1235 (2000).

[3] R. Schor and Michael O'Carroll, J. Stat. Phys. 99, Nos. 5/6, 1265-1279 (2000).

[4] R. Schor and Michael O'Carroll, J. Stat. Phys. 109, Nos. 1/2, 279-288 (2002).

[5] P.A. Faria da Veiga, M. O’Carroll and R. Schor, Phys. Rev. E66, 027108, 1-4 (2002).

[6] P.A. Faria da Veiga, L. Ioriatti and M. O'Carroll, Phys. Rev. E66, 016130, 1-9 (2002).

[7] P.A. Faria da Veiga and M. O'Carroll, in Workshop on Integrable Theories, Solitons and Duality, IFTUnesp, July 1-6, 2002, L.A. Ferreira, J.F. Gomes e A.H. Zimerman eds., Journal of High Energy Physics, February (2003). Available from http://jhep.sissa.it .

[8] P.A. Faria da Veiga, M. O'Carroll, E. Pereira and R. Schor, Commun. Math. Phys. 220, 377-402 (2001).

[9] D. Brydges, J. Fröhlich, T. Spencer, Commun. Math. Phys. 83, 123-150 (1982).

[10] J. Glimm and A. Jaffe, Quantum Physics: A Functional Integral Point of View (Spriger Verlag, New York, 1986).

[11] B. Simon, Statistical Mechanics of Lattice Gases (Princeton University Press, Princeton, 1994).

[12] R. Schor, Commun. Math. Phys. 59, 213-233 (1978).

[13] P.A. Faria da Veiga, M. O’Carroll, and R. Schor, Phys. Rev. D67, 017501 (2003).

[14] P.A. Faria da Veiga, M. O'Carroll, and R. Schor, Phys. Rev. D (2003), to appear.

[15] M. Reed and B. Simon, Modern Methods of Mathematical Physics, vol. 4, Analysis of Operators (Academic Press, New York, 1978)

[16] M. Reed and B. Simon, Modern Methods of Mathematical Physics, vol. 2, Fourier Analysis, SelfAdjointness (Academic Press, New York, 1972) 